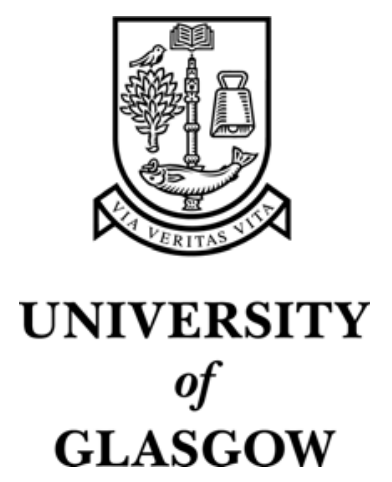

Babamahmoodi, F. and Aghabarari, F. and Arjmand, A. and Ashrafi, G.H. (2006) Three rare cases of anthrax arising from the same source. Journal of Infection 53(4):e175-e179.

http://eprints.gla.ac.uk/3098/ 


\section{THREE RARE CASES OF ANTHRAX ARISING FROM THE SAME SOURCE}

Farhang Babamahmoodi ${ }^{1}$, Fereshteh Aghabarari ${ }^{1}$, Abbas Arjmand ${ }^{1}$ and G.Hossein Ashrafi $^{2 *}$

${ }^{1}$ Department of Infectious Disease, Sari School of Medicine, Mazandaran University of Medical Sciences, 48168 Sari, Iran

${ }^{2}$ Division of Pathological Sciences, Institute of Comparative Medicine, University of Glasgow, Bearsden Road, Glasgow, UK, G61 - 1QH,

Tel: 00441413302874

Fax: 00441413305602

Email: h.ashrafi@vet.gla.ac.uk

* Corresponding author

Number of Figure: 1

Number of tables: 0

Key words: Anthrax, Gastrointestinal, Oropharyngeal, Intestinal, Meningitis

Running title: Cases of anthrax in Northern Iran 


\section{$\underline{\text { Abstract }}$}

Anthrax is an acute bacterial infection caused by Bacillus anthracis. Humans become infected under natural conditions by contact with infected animals or contaminated animal products. About $95 \%$ of human anthrax is cutaneous and 5\% respiratory. Gastrointestinal anthrax is very rare, and has been reported in less than $1 \%$ of all cases. Anthrax meningitis is a rare complication of any of the other three forms of disease. We report three rare cases of anthrax (gastrointestinal, oropharyngeal and meningitis) arising from the same source. The three patients were from a single family and were admitted with different clinical pictures after the ingestion of half-cooked meat from a sick sheep. These cases emphasize the need for awareness of anthrax in the differential diagnosis in areas where the disease remains endemic.

\section{$\underline{\text { Introduction }}$}

Anthrax is a zoonotic infection, the causative agent of which is Bacillus anthracis. Ingestion or handling of the infected animal meat or other products can transmit the disease (1). B. anthracis is non-motile, rod like, gram-positive aerobic bacillus that produces central oval-shaped spores. It is characterized by rough, irregular and often comma-shaped colonies in blood agar medium. The mature spores can persist for years in dry soil but are destroyed by boiling in water for ten minutes (2). Grazing animals often swallow these spores which develop into the encapsulated vegetative mature bacilli in the circulation (3). B. anthracis is an extra cellular pathogen that can avoid phagocytosis. Anthrax toxin contains three proteins: PA (protective antigen), EF (edema factor) and LF (lethal factor). These factors are effective in decreasing the effect of polymorphonuclear function (3). Anthrax is a zoonotic disease with a worldwide distribution. It can be transmitted from animal to animal or from animal to humans, however, no human to 
human transmission has been documented. Humans are incidentally infected by coming into contact with contaminated animals or animal products (4). The form of the disease depends on the route by which spores enter the body i.e. a skin lesion, inhalation, or ingestion (5). In cutaneous anthrax, exposed areas of skin such as arms, hands, head or neck are commonly affected. The organisms are inoculated through an abrasion in the skin. The primary skin lesion appears as a painless, pruritic papule 3 to 5 days after exposure. The papule then enlarges in one to two days to form an ulcer. The ulcer is usually 1-3 cm in diameter with round, regular borders. Vesicles may surround the ulcer over the next several days, necrosis and drying of the ulcer lead to the characteristic black eschar (7).

Inhalational anthrax is a deadly form of the disease caused by the inhalation of endospores. Initial symptoms resemble a simple upper respiratory infection for the first 13 days. The patients develop malaise, fatigue, myalgias, non productive cough and fever. The second phase of the disease is complicated by acute dyspnea, stridor and hypoxemia. Physical examination of a patient with inhalational anthrax is most remarkable for moist, crepitant crackles in the lungs pleural effusions and marked widening of the mediastinum. The lung parenchyma is usually normal in appearance, but pneumonia may be present (7). Gastrointestinal anthrax occurs in two different clinical forms, oropharyngeal and intestinal. The incubation period for both forms is typically between 1-6 days (8). Oropharyngeal anthrax is an uncommon variant of GI disease (7). It is characterized by a temperature higher than $39^{\circ} \mathrm{C}$, ulcers in posterior oropharynx (pseudomembranous ulcerations), severe sore throat, dysphagia and regional lymphadenopathy with marked neck swelling (8). This form often has a more favorable prognosis. However, some patients die from respiratory distress or sepsis (7). 
Gastrointestinal anthrax usually appears following ingestion of inadequately cooked contaminated meat. The primary infection starts when the spores are ingested and passed through the stomach. Ulcerative intestinal lesions are usually accompanied by hemorrhagic lymphadenitis. The signs are quite variable and include fever, anorexia, vomiting, abdominal pain, hematemesis, severe bloody diarrhea and in some cases, severe progressive ascites. The incubation period has been reported differently from 2-5 days. The diagnosis is rarely made before death except in endemic areas $(9,10,11)$. Anthrax meningitis is a rare complication of the three other forms of the disease. Meningeal infection results from hematogenous or lymphatic spread of the bacilli to the CNS; symptoms include nuchal rigidity, fever, headaches, seizures, agitation and delirium. Pathologic findings are hemorrhagic meningitis, bloody CSF, containing inflammatory infiltrates and large numbers of bacilli (7). In this article we report three rare cases of anthrax arising from the same source.

Background: A Family in Agha Mashad village (Mazandaran province, northern Iran) had five sheep and one appeared unwell unexpectedly. The meat from the sick animal was eaten by the family resulting in three family member falling ill and being admitted to hospital. When the remaining four sheep become sick of the same condition some time later, their meat was distributed throughout the village. After diagnosis of the initial case, meat was gathered from the villagers and analyzed in the laboratory, confirming the presence of B. anthracis in all samples.

\section{$\underline{\text { Patient 1: }}$}

A 34-year-old female was admitted to the Intensive Care Unit (ICU) section of Razi hospital, Ghaemshahr (Mazandaran Province, Iran) with high temperature, vomiting and a low level of consciousness $(\mathrm{GCS}=5)$. She had a history of fever, generalized abdominal 
pain, nausea and vomiting which had developed over 48 hours after the ingestion, 5 days previously, of half-cooked meat from a sick sheep.

Weakness, progressive headache and gradually decreasing consciousness have respectively been added to the picture one day before admission. She was not on any medication before her illness, and the following results were obtained in the physical examination at her admission:

Temperature: $38^{\circ} \mathrm{C}$, a regular pulse rate of $110 / \mathrm{min}$, blood pressure: $110 / 70 \mathrm{mmHg}$, respiratory rate: 33/min, miotic pupils, buccal mucosa dehydrated, without lymphadenopathy or mucocutaneous ulceration.

Heart: normal, lung: normal apart from tachypnea, soft abdomen without organomegaly without ascites, upper and lower limbs: normal, ophthalmoscopy: normal. Anthrax meningitis was clinically suspected from an anthrax endemic area. Laboratory examinations were requested and the following results were obtained: The patient had a Hb of 10.5 gr/dl, WBC: $21300 / \mu 1$ (poly: $82 \%$, lymph: 13\%, band: $5 \%$ ), Platelets: $134000 /$ $\mu 1$. Other coagulation tests were normal, as were kidney function tests. Liver function tests (AST, ALT) increased to five times the normal upper value, stool exam, urine analysis and urine culture were normal, chest x-ray and ECG were not remarkable.

Cerebrospinal fluid examination showed RBC: many, WBC: $3650 / \mu 1$ (poly: 93\%, lymph: 7\%), protein: $50 \mathrm{mg} / \mathrm{dl}$ and sugar: $165 \mathrm{mg} / \mathrm{dl}$ (the blood sugar was $335 \mathrm{mg} / \mathrm{dl}$ at the same time).

Cerebrospinal fluid smears showed a Gram-positive bacillus (B. anthracis) infection. Blood culture was positive after 2 days and showed Gram-positive bacilli (B. anthracis) (Figure 1). Unfortunately, despite treatment with IV penicillin G 24000000 U/day and all necessary supportive measures in ICU, the patient died 3 days after admission with a 
picture of decreased platelet, prolonged prothrombin time, increased fibrin degradation product and disseminated intravascular coagulation.

\section{Patient 2:}

A 12-year-old boy was admitted to the infectious diseases ward of Razi hospital, Ghaemshahr along with his mother (the first patient) and history of generalized abdominal pain, fever, nausea, anorexia, vomiting and diarrhea, developed 72 hours after ingestion of half-cooked meat (5 days before admission) from a sick sheep. He did not have a history of any known disease and was not on any medication before his illness. The following results were obtained in the physical examination at his admission:

Temperature: $39 / 2^{\circ} \mathrm{C}$, pulse rate: $90 / \mathrm{min}$ and regular, blood pressure: $120 / 80 \mathrm{mmHg}$ and respiratory rate: $22 / \mathrm{min}$. The patient was completely oriented but with a toxic picture, buccal mucosa dehydrated, coated tongue, without lymphadenopathy and meningeal signs, heart and lungs were normal. There was a distended abdomen with mild generalized tenderness and ascites with no organomegaly, normal bowel sounds. The stool was not bloody in rectal examination.

Intestinal anthrax was suspected on clinical grounds (following consideration of the history and clinical signs) and laboratory examination were requested. The following results were obtained; Hgb: $15.1 \mathrm{gr} / \mathrm{dl}$, WBC: 17300/ $\mu$ l (poly: 80\%, lymph: 16\%, band: 2\%, mono: $2 \%$ ), platelet: $227000 / \mu 1$, coagulation tests, kidney and liver function tests were normal. In stool exam: RBC: 12-14/hpf, WBC: 6-8/hpf, and stool parasite was negative, urine analysis was normal, urine culture was negative, chest x-ray: not remarkable, plain abdominal x-ray revealed gaseous distension and multiple fluid levels, and abdominal sonography showed ascites (2000cc). Penicillin G 12000000 U/day was administered intravenously for two weeks. The patient was discharged in good general condition and completely recovered after two weeks. 


\section{Patient 3:}

A 14-year-old young adult female was admitted to infectious disease ward of Razi hospital, Ghaemshahr with a history of fever, sore throat, dysphagia, hoarseness, marked and painful swelling of the left side of the neck which had developed 72 hours after the ingestion of half- cooked meat ( 8 days before admission) from a sick sheep.

She did not have a history of any known disease and she was the sister of patient 1 and aunt of patient 2. She was not on any kind of medication before her illness and the following results were obtained in the physical examination at her admission:

Temperature: $39.4{ }^{\circ} \mathrm{C}$, pulse rate: $98 / \mathrm{min}$ and regular, blood pressure: $120 / 80 \mathrm{mmHg}$, respiratory rate: $20 / \mathrm{min}$.

The left tonsil was edematous, congested and enlarged, mouth lesions were present on the left tonsil, which was covered by a grey pseudo membrane. Painful swelling, enlargement and tenderness of the lymph nodes of the left side of the neck were notable. Heart, lung and abdomen were normal. Considering the history and clinical manifestations of the patient, oropharyngeal anthrax was suggested clinically and laboratory examinations were requested with following results:

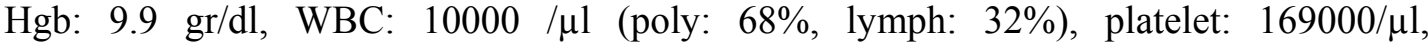
coagulation tests, kidney and liver function tests, stool exam, urine analysis and urine culture were normal, chest x-ray: not remarkable. The main diagnostic test was a throat smear of the lesion of the left tonsil, which showed Gram - positive bacilli. Penicillin G $16000000 \mathrm{U} /$ day was accordingly administered intravenously. The patient responded well with the treatment and was discharged in good general condition and complete recovery after two weeks. 


\section{$\underline{\text { Discussion }}$}

Anthrax has been an important disease throughout history (12). Currently, very few cases of anthrax occur naturally in developed countries (4). The incidence of infection has been reduced dramatically by the vaccination of highrisk people and animals, along with improvements in industrial hygiene (4). Anthrax spores remain endemic in rural regions of the world, such as Africa and Asia where vaccination programs are difficult to implement (4). Of the three forms of the disease, inhalational anthrax has the highest mortality rate $(\geq$ $40 \%$ with antibiotic treatment (13) and $97 \%$ without antibiotic treatment) followed by gastrointestinal (GI) anthrax ( $\leq 40 \%$ with antibiotic treatment) (14) and cutaneous anthrax (1\%with antibiotic treatment and 10\%-20\% without antibiotic treatment) (15). In the outbreak with the largest reported number of oropharyngeal anthrax cases $(n=24)$, oral lesions were located on the tonsil in $72 \%$ of the cases (16), neck swelling was present in all patients but unilateral in $75 \%$, lymph nodes measuring up to $4 \mathrm{~cm}$ in diameter were noted (16). Positive cultures from oropharyngeal swabs have been reported for some patients with oropharyngeal anthrax (16). The third patient introduced in this report had exactly the same picture. In a review of surveillance data from Sivas region of Turkey the presence of Gram-positive bacilli was demonstrated in a smear of oropharyngeal lesions for 6 out of 6 patients and B. anthracis was isolated on culture, but all blood cultures were negative (14) These data suggest that oropharyngeal culturing is important in the diagnosis of B. anthracis. Blood cultures should be obtained from all patients with suspected anthrax infection. However, since the vegetative from of most strains of B. anthracis is sensitive to a wide variety of antibiotics, treatment with antibiotics reduces the probability of successfully culturing the organism from blood (13). Confirmed epidemic cases of GI anthrax have often been reported in developing countries (17). 
In GI anthrax abdominal pain can become so severe that patients present the signs of acute abdomen with rebound tenderness (6). Abdominal radiographs in this form provide nonspecific findings suggestive of obstruction, such as gaseous distension and multiple fluid levels (10) evidence of ascites (18). The clinical symptoms observed in our second case were the same as reported in the texts. B. anthracis has been isolated from stool specimens from several animal species and humans (8). Since the utility of stool cultures in diagnosis of $B$. anthracis infections in humans remains uncertain, blood or ascitic fluid for culture and possibly for PCR should be obtained from patients with the disease (14). Because of the propensity of B. anthracis infection of the gastrointestinal tract to progress to sepsis or death, both types of gastrointestinal anthrax should be treated aggressively as a systemic illness (14). Most of the patients who died from anthrax sepsis, despite treatment, had incubation periods of less than 48 hours and typically died within 48 hours of the onset of symptoms. This happened to the first case reported here despite treatment under ICU. This suggests that antibiotic treatment should be initiated as soon as possible for maximum efficacy (19). In view of the rapid progression of GI anthrax and its high mortality rate, even with therapy, physicians must be alert for history taking especially in the patients with gastrointestinal bleeding (3). More men have been reported to contract intestinal or oropharyngeal anthrax compared to women. This may be related to exposure to $B$. anthracis during the butchering of infected animals, which is usually done by men (16). Meningitis caused by B. anthraces is almost always fatal within 1-6 days, even with antibiotic therapy (7). Children tend to have a more fulminating course than adults (19). Most infections in areas where anthrax is endemic occur in adults but this may be a function of exposure (19). Awareness of anthrax in a differential diagnosis remains important in disease endemic areas for prevention. Workers exposed to contaminated animal products in high-risk industries should be vaccinated against B. anthracis. Anthrax 
in animals can also be controlled by the use of a live attenuated vaccine. In this article we report three rare cases of anthrax (gastrointestinal, oropharyngeal and meningitis) arising from the same source in northern Iran (Mazandaran province). The three patients were from a single family and were admitted with different clinical pictures after the ingestion of half-cooked meat from a sick sheep.

These cases are very notable, because all three rare pictures of anthrax occurred in the same family and emphasize the need for awareness of anthrax in differential diagnosis in an area where the disease remains endemic.

\section{Acknowledgments}

We would like to thank Professors M Saveria Campo and David Taylor for critically reading the manuscript. This work was supported by Mazandaran University of Medical Sciences, Iran.

\section{$\underline{\text { References }}$}

1. Davies JCA: A major epidemic of Anthrax in Zimbabwe. Cent Afr. $j$ Med 1982; 28: 291-8.

2. Gleiser CA: Pathology of Anthrax infection in animal hosts. Fed Prac 1967; 26(5):1518-1521.

3. Brachman Ps, Kaufmann AF: Anthrax In: Evans As, Brachman Ps, Eds. Bacterial infections of humans. New York: Plenum Medical Book Company 1998; 95-111.

4. Dixon T. Anthrax Medical progress, Review article .N Engle j.Med 1999; 341: 815-26.

5. Mckendrick DR, Anthrax and its transmission to humans. Cent Afr Med. 1980; 26: 126129.

6. Tekin A, Bulut N, Unal T: Acute abdomen due to anthrax. Br J surg 1997; 84: 813. 
7. Meselson M, Guillemin J, Hughjones M, The sverdlovsk Anthrax outbreak of 1979. Science 1994; 266: 1202-8.

8. Lakshmi N, Kumar AG. An epidemic of human anthrax a study. Indian J Pathol Microbial. 1992.35: 1-4.

9. Khhott E, Sehat A, Ashraf M: Anthrax, a continuous problem in south west in IRAN, The American, J of Medical Sci 1964; 566-575.

10. Jena Gp: Intestinal Anthrax in man: a case report. Center Afr, J, Med. 1980; 26: 253 4.

11. Daniel P: lew Bacillus Anthracis in: mandell GL, Bannett JE.

Dolin R (Eds) Mandell, Douglas and Bennett principles and practice of infectious Disease, 5th ed, Churchill living stone, 2000, 2215- 20.

12. Mandell. Principles and practice of infectious, 5th ed. In .Iew Dp, ed Churchill Livingstone, Inc. 2000: 2215-20.

13. Jernigan JA, Stephens Ds, Ashford DA, al, Bio terrorism - related inhalational anthrax the first 10 cases reported in the United States, Emerge infect Dis. 2001; 7: 933- 944.

14. Doganay M, Almac A, Hanagasi R. Primary throat anthrax. Scand J infects Dis. 1986; 18: $415-419$.

15. His Majesty's stationery office, Report of the Departmental committee Appointed to inquire as to precautions for preventing danger of infection from Anthrax in the manipulation of wool, Goat, Hair. And camel Hair, London, England: His Majesty's stationary office; 1918.

16. Siris Anthana T. Outbreak of oral oropharyngeal anthrax: an unusual manifestation of human infection with Bacillus anthracis. AmJ, Trop Med Hyg. 1984: 33: 144- 150.

17. Randall K: Holmes, Anthrax In: Braunval, Fauci, Kasper, Longo, Jamson. Editors, Harrison's Principles of internal Medicine, $15^{\text {th }}$ ed. New Yoirk .Mc Graw - Hill Inc, 2001; 
$914-15$.

18. Dutz W, Said F, Kohout E. Gastric Anthrax with massive ascites, Gut. 1970; 11: $352-$ 354.

19. Ndybahinduke DGK. An outbreak of human gastrointestinal anthrax. Ann its super sanita. 1984; 20: $205-208$ 


\section{Figure Legend}

Figure 1. B. anthracis in the blood culture. Blood was cultured for 2 days and showed $B$. anthracis (arrows). 
Babamahmoodi et al. Figure 1

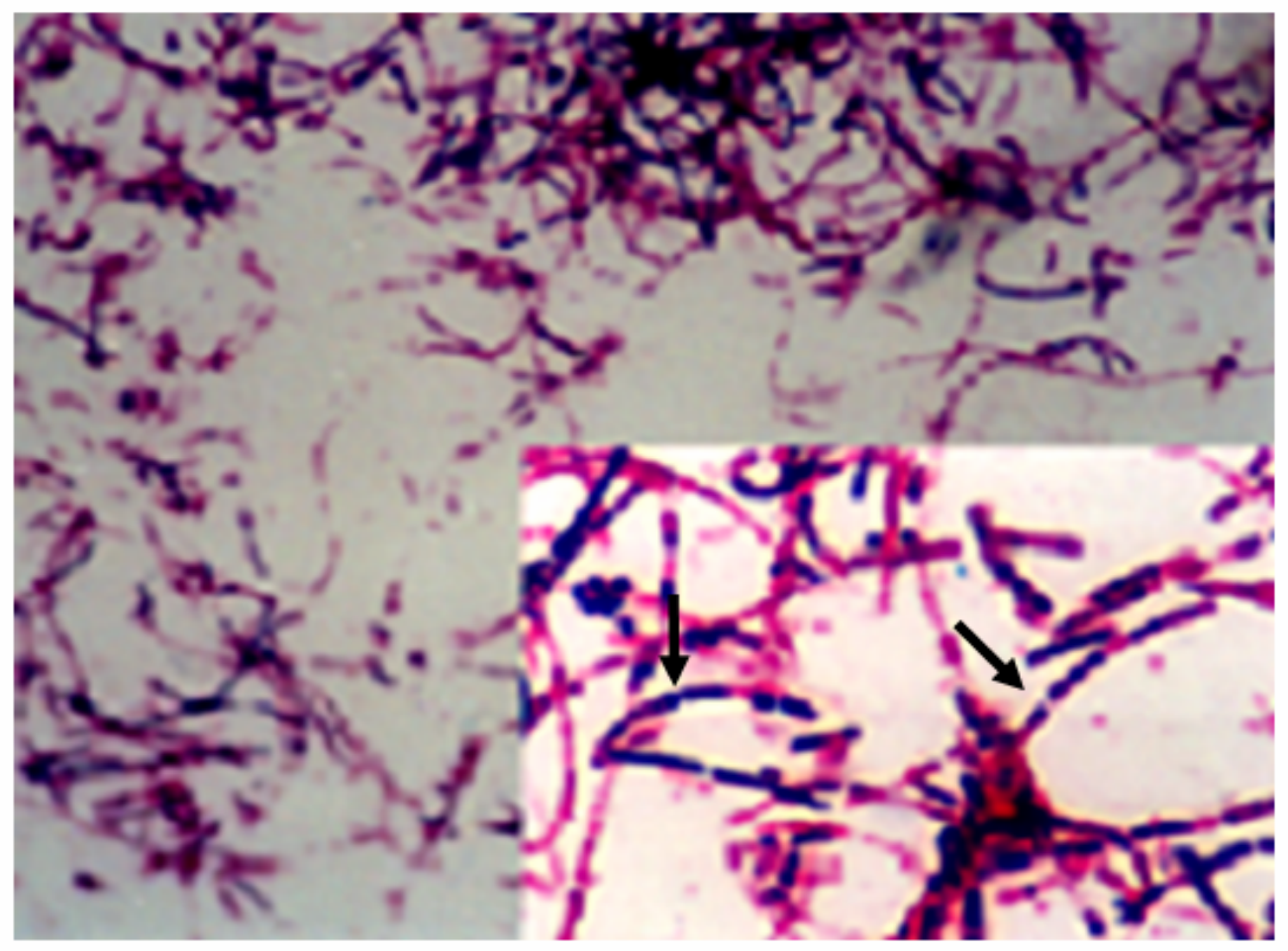

